\title{
Welche Voraussetzungen muss ich für diese GOP erfüllen?
}

\section{Dr. med. A.F., KVB, Oberpfalz:}

Ab 1.4.2011 gibt es neue Gebührenordnungspositionen (GOP) 01425 und 01426 für die Versorgung von Palliativpatienten. Ich betreue derzeit drei solcher Patienten. Welche Voraussetzungen muss ich erfüllen, um diese GOP abrechnen zu können?

Antwort: Als Hausärztin gehören Sie zu einer grundsätzlich qualifizierten Facharztgruppe, die Palliativpatienten betreuen kann. Für die spezialisierte ambulante Palliativversorgung (SAPV) von Palliativpatienten muss allerdings ein entsprechend qualifiziertes Palliativteam zur Verfügung stehen. Zur Zeit ist dies jedoch flächendeckend in Bayern noch nicht überall vorhanden.

Die Einbindung der SAPV ist an bestimmte medizinische Voraussetzungen gebunden: Es muss mindestens eines der nachstehenden Kriterien erfüllt sein:

- ausgeprägte Schmerzsymptomatik,

- ausgeprägte neurologische/psychiatrische/psychische Symptomatik,

- ausgeprägte respiratorische/kardiale Symptomatik,

- ausgeprägte gastrointestinale Symptomatik,

- ausgeprägte ulzerierende/exulzerierende Wunden oder Tumoren,

- ausgeprägte urogenitale Symptomatik. Liegt eine solche Situation vor, wird die Verordnung von SAPV auf dem Vordruck Muster 63 „Verordnung Spezialisierter ambulanter Palliativversorgung (SAPV)“ vorgenommen. Über die SAPV werden dann entsprechend

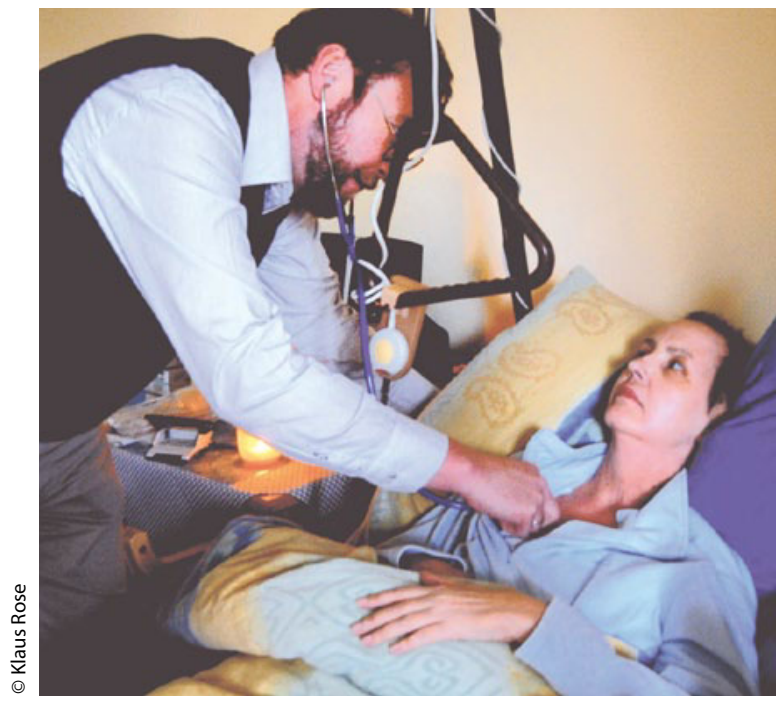

Der erhöhte Betreuungsaufwand bei der ambulanten Sterbebegleitung wird in Bayern extra honoriert.

dem jeweiligen aktuellen Versorgungsbedarf - je nachdem, was der Hausarzt als notwendig oder sinnvoll erachtet - folgende Leistungen erbracht:

Beratungsleistungen für den Patienten und/ oder den betreuenden Hausarzt, Koordination der Versorgung, additiv unterstützende Teil- oder auch vollständige Versorgung des Palliativkranken.

Voraussetzung ist, dass der Patient mit gesicherter Diagnose "Palliativbehandlung" (Z 51.5) kodiert werden kann. Die Verordnung muss der Versicherte durch Vorlage bei seiner Kasse genehmigen lassen (wie bei Verordnung Häuslicher Krankenpflege). Diese übernimmt erst einmal bis zu ihrer endgültigen Entscheidung über die weitere Leistungserbringung die Kosten für die verordneten und erbrachten Leistungen, wenn die Verordnung spätestens an dem dritten der Ausstellung folgenden Arbeitstag der Kasse vorgelegt wird.

Seit dem 1.1.2010 wird in Bayern eine gesonderte Vergütung für den erhöhten Betreuungsaufwand bei Patienten in der ambulanten Sterbebegleitung für alle Vertragsärzte gezahlt. Bayerns Ärzte erhalten zusätzlich zum Honorar für ihre ärztlichen Leistungen eine Pauschale von $20,00 €$ pro Besuch eines Palliativpatienten. Dazu wird in Bayern die GOP 97005 einmal im Quartal beim ersten Besuch angesetzt. Ab diesem Zeitpunkt wird die GOP automatisch für alle Folgebesuche angesetzt und jeder weitere Besuch entsprechend mit Zuschlag vergütet.

Die Pauschale wird für die EBM-Leistungen 01 410, 01 411, 01 412, 01 413, 01 415, inklusive Nachtbesuche gezahlt. Wichtig für den Hausarzt ist, dass seine bisherige Tätigkeit im gewohnten Umfang parallel weiter laufen kann. Sofern Hausbesuche, Beratungen und berechenbare Sonderleistungen durchgeführt werden, rechnet der Hausarzt diese nach dem EBM über die KV ab. 